\title{
Many-to-Many Communication: A New Approach for Collaboration in MANETs
}

\author{
Renato M. de Moraes \\ Dept. of Computing Systems \\ University of Pernambuco \\ Recife, PE 50720-001, Brazil \\ Email: renato@dsc.upe.br
}

\author{
Hamid R. Sadjadpour \\ Dept. of Electrical Engineering \\ University of California (UCSC) \\ Santa Cruz, CA 95064, USA \\ Email: hamid@soe.ucsc.edu
}

\author{
J.J. Garcia-Luna-Aceves \\ Dept. of Computer Engineering at UCSC and \\ Palo Alto Research Center \\ 3333 Coyote Hill Rd., Palo Alto, CA 94304, USA \\ Email: jj@soe.ucsc.edu
}

\begin{abstract}
We introduce a collaboration-driven approach to the sharing of the available bandwidth in wireless ad hoc networks, which we call many-to-many cooperation, that allows concurrent many-to-many communication. This scheme is based on the integration of multi-user detection and position-location information with frequency and code division in mobile ad hoc networks (MANETs). Transmissions are divided in frequency and codes according to nodal locations, and successive interference cancellation (SIC) is used at receivers to allow them to decode and use all transmissions from strong interfering sources. Consequently, the interference is divided into constructive interference (COI) and destructive interference (DEI). We show that, if each node is allowed to expand its bandwidth, both the link's Shannon capacity and the per source-destination throughput scale like $O\left(n^{\frac{\alpha}{2}}\right)$ (upperbound) and $\Omega[f(n)]$ (lower-bound), for $n$ nodes in the network, a path loss parameter $\alpha>2$, and $1 \leq f(n)<n^{\frac{\alpha}{2}}$. Many-to-many cooperation allows multi-copy relaying of the same packet, which reduces the packet delivery delay compared to single-copy relaying without any penalty in capacity.
\end{abstract}

\section{INTRODUCTION}

The protocol stacks of wireless ad hoc networks implemented or proposed to date have been designed to try to avoid interference. Hence, communication protocols used in wireless ad hoc networks today are meant to support reliable communication among senders and receivers that are competing with one another for the use of the shared bandwidth. This "competitiondriven" view of bandwidth sharing has had profound implications on network architectures and methods used to access the channel and disseminate information. Gupta and Kumar [1] showed that, in a wireless connected network with static nodes, the throughput for each node degrades as the number of nodes increases under the competition-driven view of networking. That is, it scales as $\Theta(1 / \sqrt{n \log (n)}),{ }^{1}$ where $n$ is the number of nodes in the network.

Grossglauser and Tse [2] analyzed a two-hop, single-relay forwarding scheme for MANETs in which a source passes a packet to a relay that in turn delivers it to the destination when the two nodes are close to each other. This and many subsequent studies on how to make MANETs scale by using mobility [2], [3], [4], [14] consider each transmission as competing with all the other concurrent transmissions in the network. However, because a relay cooperates with a source by storing the source's packet until it is close enough to the intended destination, the throughput of MANETs can be increased. ${ }^{2}$

Recently, Toumpis and Goldsmith [5] have shown that the capacity regions for ad hoc networks are increased when multiple

\footnotetext{
${ }^{1} \Omega, \Theta$ and $O$ are the standard order bounds. $\log (\cdot)$ is the natural logarithm.

${ }^{2}$ In [2], the per source-destination throughput scales as $\Theta(1)$.
}

access schemes are combined with spatial reuse (i.e., multiple simultaneous transmissions), multihop routing (i.e., packet relaying), and SIC, even without performing power control. Also, SIC circuits with simple implementation and low complexity have been introduced [6], and code division multiple access (CDMA) [7] and global positioning system (GPS) [8] technologies have been already integrated into a single IC chip [9]. Although CDMA and SIC for ad hoc networks have been studied in the past [10], [11], [12], [13], prior approaches have assumed that each transmission competes with others.

These works [1], [2], [3], [5], [10], [11], [12], [13], [14], characterize a one-to-one communication approach which stems from cellular concepts and in our opinion, it is not appropriate for ad hoc networks. Our earlier work [4] describes a setting for one-to-many communication. In this scenario, a node relays its packet to multiple relay nodes that are close, allowing them to cooperate to search for the destination. In this scheme, however, all the transmitting nodes in each communication session compete with each other to transmit their packets. Ghez et al. [15] and Tong et al. [16] explain a framework for many-toone communication. In this context, multiple nodes cooperate to transmit their packets simultaneously to a single node using CDMA and the receiver node utilizes multiuser detection to decode multiple packets. Under this condition, two groups of multiple transmitting nodes that are close to each other have to compete with one another to transmit their packets to their respective receivers.

From the above results, it appears that a cooperative scheme to bandwidth sharing is not only desirable for attaining more scalable MANETs, but feasible in practice. In this paper, we present an integrated approach to cooperative bandwidth sharing in MANETs and propose what we call many-to-many cooperation. Many-to-many cooperation is a vision for multiple concurrent communication settings (i.e., a many-to-many framework). In this scheme, nodes access the available channel(s) and forward information across a MANET in such a way that concurrent transmissions become useful at destinations or relays. Our cell size limits the number of nodes in each cell, on average, making it feasible to decode the dominant interference using multiuser detection. Hence, sender-receiver pairs collaborate, rather than compete, and the adjacent transmitting nodes with strong interference to each other are no longer an impediment to scaling laws but rather an acceptable communication by all receiving nodes for detection and relaying purposes. A consequence of such a strategy is an increase in the receiver complexity of all the nodes in the network. 
We show that, by utilizing mobility [2], multiuser diversity ${ }^{3}$ [17], SIC, cognition ${ }^{4}$ and bandwidth expansion, the link's Shannon capacity and the per source-destination throughput attain an upper-bound of $O\left(n^{\frac{\alpha}{2}}\right)$ and a lower-bound of $\Omega[f(n)]$, for $n$ total nodes in the network, a path loss parameter $\alpha>2$, and $1 \leq f(n)<n^{\frac{\alpha}{2}}$.

Section II summarizes the basic network model that has been used recently to analyze the capacity of wireless networks [1], [2], [3], [4], [11], [14]. Section III describes the details of many-to-many cooperation. Section IV presents the the link's Shannon capacity, the per source-destination throughput, and the bandwidth requirement. Section V compares our approach with previous schemes, showing that with similar bandwidth expansion, our approach outperforms other existing techniques. Section VI concludes the paper.

\section{NeTWORK MODEL}

The term cell denotes the set of nodes located inside a defined area of the network. The receiver range ${ }^{5}$ of a node is defined as the radius, measured from the node, which contains all other nodes of the same cell. The cluster associated with a given node is the set of cells reached by the receiver range of this node.

Our assumptions are consistent with prior work [1], [2], [3], [4], [11], [14]. Also, in this paper, nodes are considered to have SIC capability. The modeling problem we address is that of a MANET in which $n$ mobile nodes move in a unit square area. We assume that cells have square shapes, each with area equal to $a(n)=\frac{1}{\phi n}$, in which $\phi \in(0,1)$ is the cell area parameter of the network, as illustrated in Fig. 1. We consider that the communication occurs only among those nodes that are close enough (i.e., in same cell), so that interference caused by farther nodes is low, allowing reliable communication. In other words, the receiver chooses the closest nodes because they present the best channel, in a respective order, due to the assumption of the simple path propagation model, i.e., the receiver takes advantage of multiuser diversity [17]. Our model resembles the one introduced by Grossglauser and Tse [2], who consider a packet to be delivered from source to destination via one-time relaying. The position of node $i$ at time $t$ is indicated by $X_{i}(t)$. Nodes move according to the uniform mobility model [3], in which the steady-state distribution of the mobile nodes is uniform.

Each node simultaneously transmits and receives data during a communication time period, through different frequency bands, since each data link is assumed half-duplex. ${ }^{6}$ This period of communication is called a communication session. Furthermore, each session is divided into two parts. A neighbor discovery protocol is used by nodes during the first part to obtain their neighbors information (e.g., node identification (ID)), and the transmission of data is performed during the second part. Each node has a unique ID that does not change with time, and each node can simultaneously be a source (or relay) while transmitting and a destination (or relay) while receiving, during a session. Each source node picks a single arbitrary destination to whom it sends packets.

3 i.e., a node transmits a packet to all its nearest neighbors, and those relays deliver the packets to the destinations when each destination becomes a close neighbor of each relay.

${ }^{4}$ To allow a node to know where it is and who the nodes in the same cell are.

${ }^{5}$ We adopt receiver range for a node because it is used here to distinguish constructive interference from destructive one (as described later), in contrast to the common use of transmission range as in [1].

${ }^{6}$ Half-duplex means that a node cannot transmit and receive data simultaneously through the same frequency bandwidth.

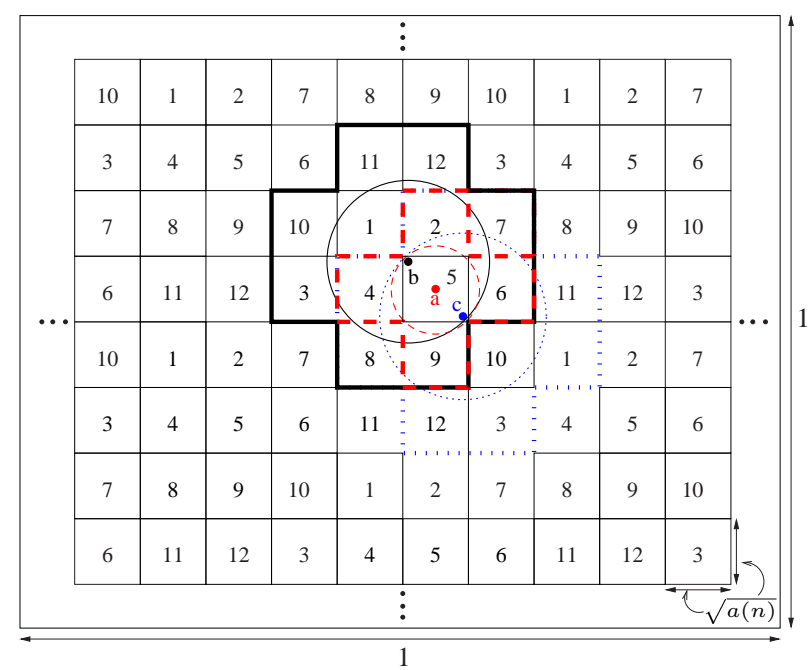

Fig. 1. Cells numbering in the unit square network. $a(n)=\frac{1}{\phi n}$ is the cell area. Each cell is associated to a control frequency bandwidth $\left(\omega_{1}\right.$ to $\left.\omega_{12}\right)$ and to a PN sequence set ( $\xi_{1}$ to $\left.\xi_{12}\right)$.

\section{MANY-TO-MANy COOPERATION}

In many-to-many cooperation, several nodes transmit concurrently to many other neighbor nodes, and all such transmissions are decoded. Thus, a node may concurrently send to and receive from many nodes. Since full-duplex data communication in the same frequency band is not practical, we present an example of how many-to-many cooperation can be implemented with a scheme based on frequency division multiple access (FDMA) and CDMA that supports many-to-many communication.

\section{A. Bandwidth Allocation and Data Packet Forwarding}

In our specific implementation of many-to-many cooperation, we use two types of channels. Control channels are used by nodes to obtain such information as the identities of strong interference sources, the data packets expected by destinations, and the state of data channels (by virtue of training sequences). Nodes employ conventional digital transceivers for the control channels. Data channels are used to transmit data taking advantage of SIC at the receivers. Thus, there are two separate transmitter (receiver) circuits in each node. One circuit is intended to transmit (receive) control packets, and the other is used to transmit (receive) data packets. Both circuits operate in different time and frequency with respect to each other.

Control (or Signaling) Channels: Each cell is allocated a control frequency band from twelve non-overlapping control frequency bands, $\omega_{1}$ to $\omega_{12}$, to enable frequency reuse while avoiding interference in the control channels from nearby cells (see Figs. 1 and 2). Each control frequency band $\omega_{i}$ has a size of $\left|\omega_{i}\right|=\Delta \omega$ for $i=1, \ldots, 12$. Hence, the total bandwidth required for the control channels is $\Delta \omega_{C}=12 \Delta \omega$.

The maximum number of cells in a cluster associated to a given node is twelve. The number of cells and the cluster shape are chosen such that if the receiver range has maximum value, i.e., almost $\sqrt{2 a(n)}$, then the receiver range reaches all these cells. Also, two cells employing the same control frequency band are kept at least $\sqrt{5 a(n)}$ units away from each other, i.e., a safe guard-zone separation, thus guaranteeing asymptotic constant non-zero signal-to-noise and interference ratio (SNIR) as $n \rightarrow \infty$ [4] in the control channel, making signaling feasible and allowing control frequency reuse. 


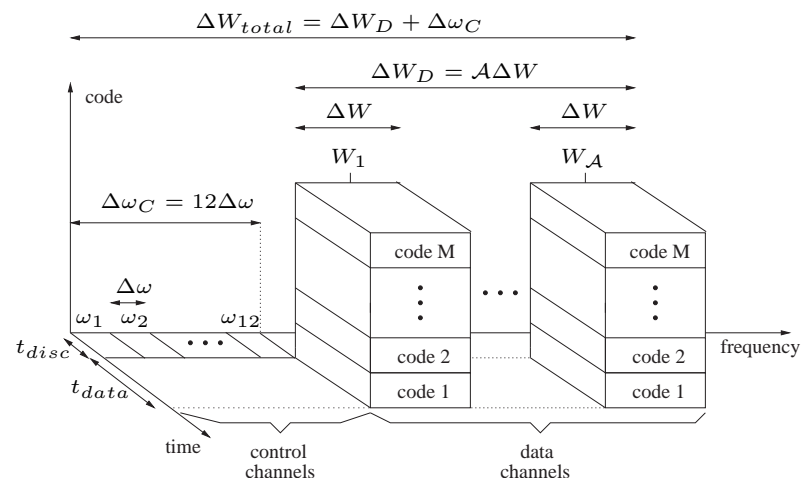

Fig. 2. Data and control channels spectra for the network.

To determine which control channel a given node should use, each node is assumed to know its own position (but not the position of any other node) by utilizing a GPS circuit [8], [9], and to store a geographical map of the cells in the network with their associated control frequencies. The GPS is also used to provide an accurate common time reference to keep all nodes synchronized.

Each node uses the control channel receiver to listen to the control channel of the cell as well as to the other 11 control channels, in order to obtain the IDs and training sequences of the other nodes in its cell and in the cluster it perceives, while not transmitting during the neighbor discovery phase.

Data Channels: To allow code reuse in the data channels while reducing the negative effects of interference, each cell is allocated a set of PN sequences (or codes) from the twelve different code sets available, $\xi_{1}$ to $\xi_{12}$, for communication in each data channel. Accordingly, each non-overlapping data channel is a half-duplex link of bandwidth $\Delta W$. If $\mathcal{A}$ is the maximum number of nodes allowed to communicate in any cell, then $\Delta W_{D}=\mathcal{A} \Delta W$ is the data bandwidth required for the entire network and $M \geq 12 \mathcal{A}$ distinct PN sequences are needed for local data communication. $M$ is also called the spreading factor (or processing gain). Also, $\Delta W=B M$, where $B$ is the original data bandwidth before spreading [7].

Because a PN sequence can be associated to a sequence of bits [7], they can be ordered and grouped as follows. $\xi_{1}=\left\{C_{1}, \ldots, C_{\mathcal{A}}\right\}, \quad \xi_{2}=\left\{C_{\mathcal{A}+1}, \ldots, C_{2 \mathcal{A}}\right\}, \ldots, \xi_{12}=$ $\left\{C_{11 \mathcal{A}+1}, \ldots, C_{12 \mathcal{A}}\right\}$, in which $C_{i}$ stands for the $i^{\text {th }} \mathrm{PN}$ sequence (or code). In this way, any set of twelve cells, numbered from 1 to 12, has a different set of codes. Therefore, by construction, the cluster seen by any node is composed of cells having distinct numbers, and consequently, different codes.

As we discuss in Section III-B, the signaling in the control channel provides each node in a cell $i$ knowledge of who the other nodes in this same cell are, and the node uses this information to choose a data channel to receive data, as well as to select a code for transmission from the available PN sequences in $\xi_{i}$ based on its own and neighbor IDs, in the following order ${ }^{7}$ : (i) The node with the highest ID in cell $i$ is associated with the data channel $\Delta W$ centered at $W_{1}$, as well as it is assigned the first PN sequence in $\xi_{i}$. (ii) The node with the second highest ID in cell $i$ is associated with the data channel $\Delta W$ centered at $W_{2}$, as well as it is assigned the second PN sequence in $\xi_{i}$, and this continues for all nodes in cell $i$. (iii) The data channels not utilized become idle in cell $i$. It happens in those cells where

\footnotetext{
${ }^{7}$ For clarity, we also indicate $W_{j}$ as the data channel associated to node $j$.
}

the number of nodes is less than $\mathcal{A}$.

In a communication session, each node only needs to know the nodes in its cell (obtained during the neighbor discovery phase) and the signal strengths received from them (by virtue of CDMA-SIC), in order to identify nodes in its receiver range. Note that nodes in a cell are very close to each other and nearfar problem is not significant here.

With the deployment illustrated in Fig. 1, two or more nodes, while moving in the same cell, can perceive clusters composed of different cells with at most twelve distinct numbers. For example, in the middle of Fig. 1, node $a$, located exactly at the center of the cell 5 , can apply SIC to decode the data signal from node $b$ and node $c$ in that same cell, each one being almost $\sqrt{a(n) / 2}$ far apart from node $a$ as shown (consequently, the receiver range for $a$ is approximately $\sqrt{a(n) / 2}$ and it is indicated by the dashed circle). Node $a$ perceives the cluster composed of the five cells $\{2,4,5,6,9\}$ indicated in dashed line (i.e., those cells reached by $a$ 's receiver range), and the other remaining closest four different cells $\{1,7,8,10\}$ are not necessary for decoding purposes. However, node $b$ has to decode signals from nodes $a$ and $c$ which is almost $\sqrt{2 a(n)}$ away (thus, the receiver range for $b$ is approximately $\sqrt{2 a(n)}$ and it is indicated by the solid circle). Hence, node $b$ perceives the cluster with all the twelve cells $\{11,12,10,1,2,7,3,4,5,6,8,9\}$ shown in solid line, i.e., those cells reached by its receiver range. Analogously, node $c$ perceives $\{2,7,4,5,6,11,8,9,10,1,12,3\}$ illustrated in dotted line. Therefore, by construction, the cluster perceived by any node is composed of cells having distinct numbers, and consequently, different codes.

At time $t$, each cell has $Z$ nodes such that the data communication is $Z$-to- $Z$, i.e., many-to-many communication (see Fig. 3 ), where $Z$ is a random variable due to mobility. Each node employs a multi-user transmitter DS-CDMA [7] (i.e., it transmits up to $Z-1$ simultaneous data packets per session in which, due to FDMA, each packet is sent through a different data channel, as illustrated in Fig. 3(downlink)), spreading the data using the PN sequence associated to its ID. The node can transmit a different data packet in each channel or choose to send the same data packet in all (non-idle) channels, or a combination of both, depending on the fact that the node has packet for any destination in the same cell it is located. Thus, multi-copies of the same packet can be simultaneously relayed to reduce delay [4].

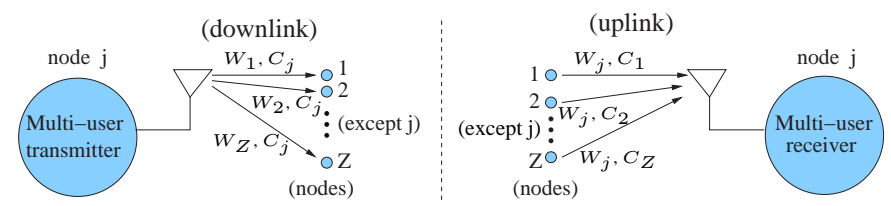

Fig. 3. Downlink and uplink description for data channels in a cell. Communication is $Z$-to- $Z$ (i.e., many-to-many).

Given that each node is endowed with a multi-user detector (the SIC circuit) for its associated receiving data channel, it is able to decode the $Z-1$ simultaneous transmissions from all nodes in its cell (see Fig. 3(uplink)).

Data packet forwarding consists of two phases [2], [4]: The packet is transmitted from the source to possibly several relay nodes during Phase 1 (i.e., multi-copies can be forwarded), and it is delivered later to its destination by only one of the relay nodes during Phase 2. Both phases occur concurrently, but Phase 2 has priority in all communications. These multiple 
one-time relays for the same packet provide better delay performance since the copies of the same packet follow different random routes, looking for the destination, reducing delay [4].

\section{B. Channel Access}

Access to the channel is controlled by the signaling that takes place over the control channels. Such signaling occurs simultaneously in all cells, without suffering high interference from each other because of the different frequency assignment and consequent safe guard-zone separation (see Section III-A).

The signaling among the nodes in the same cell must be oneto-many and cannot assume knowledge of who the nodes in a cell are, because nodes are mobile. Each node needs to inform the other nodes in its present cell about its own presence in the cell, plus other control information. From Fig. 4, access to the channel is divided in time into a discovery phase and a data-transmission phase. The period of "neighbor discovery" $t_{d i s c}$ and the period for transmission of data $t_{\text {data }}$ are constant and independent of the number of nodes in the network $(n)$. Together, they compose a "communication session." The common time reference for communication sessions is obtained through the GPS circuit. The values of $t_{d i s c}$ and $t_{d a t a}$ are system design parameters. $t_{\text {disc }}$ is subdivided into $N$ slots, each of length $T$. Hence, $T=\frac{t_{d i s c}}{N}$, where $N$ is a positive integer to calculate according to some given criterion as explained later.

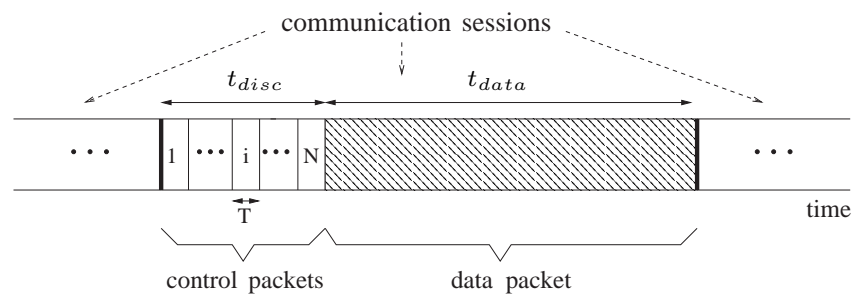

Fig. 4. Time series representation of control and data packets. $t_{\text {disc }}$ is the neighbor discovery phase. $t_{d a t a}$ is the time period for transmission of data. $t_{\text {disc }}$ plus $t_{\text {data }}$ form a communication session.

Each node simultaneously senses the channel to detect collision while transmitting in the control channel, for example, using echo cancelling techniques [18]. Accordingly, the nodes involved in a collision do not participate in that session anymore, i.e., they remain silent until the next session. Also, since only $\mathcal{A}$ codes are available per cell, then, only the first $\mathcal{A}$ nodes that successfully announced their control packets during $t_{d i s c}$ are going to transmit (or receive) data during $t_{\text {data }}$ for that session. Each control packet conveys, as a minimum, the node ID, a short training sequence and the sequence number $(\mathrm{SN})$ the node is expecting as destination, while a data packet bears long sequences of bits. Therefore, we assume that $t_{d i s c}<<t_{d a t a}$.

Each time the discovery period is about to begin, each node randomly chooses one of the $N$ mini-slots and transmits its control packet. If there is no collision, i.e., if the other nodes in the same cell choose different mini-slots to transmit, then all the other nodes in the cell will receive this packet. A collision happens every time two or more nodes in the same cell choose to transmit in the same mini-slot. Let $Z_{i}$ be the number of nodes in the same cell choosing the mini-slot $i$ to transmit their control packets. Let $Z_{\max }$ be the maximum number of nodes in any cell. The probability of collision $\mathbb{P}_{c}$ is given by

$\mathbb{P}_{c}=\mathbb{P}\left\{Z_{i} \geq 2\right\}=1-\left(1-\frac{1}{N}\right)^{Z_{\max }}-\frac{Z_{\max }}{N}\left(1-\frac{1}{N}\right)^{Z_{\max }-1} \cdot$

The criterion used to choose $N$ is as follows. We calculate $N$ such that there is no collision with probability approaching 1 as $n \rightarrow \infty$, for example, with probability $\geq 1-\frac{\log (\log (n))}{\log (n)}$. From

(1), $\mathbb{P}_{c} \leq 1-\left(1-\frac{1}{N}\right)^{Z_{\max }}$. Accordingly, we choose

$$
\mathbb{P}_{c} \leq 1-\left(1-\frac{1}{N}\right)^{Z_{\max }} \leq \frac{\log (\log (n))}{\log (n)}
$$

$\Longrightarrow N \geq\left\lceil\left[1-\left(1-\frac{\log (\log (n))}{\log (n)}\right)^{\frac{1}{Z_{\max }}}\right]^{-1}\right\rceil=N_{\min }$,

in which $\lceil x\rceil$ stands for the ceil function (i.e., the smallest integer greater than or equal to $x$ ), and $N_{\min }$ is the actual value to be implemented for $N$. Thus, we have

$$
T=\frac{t_{\text {disc }}}{N_{\min }} \text {. }
$$

The relationship between $Z_{\max }$ and $n$ is given by the following lemma, which proof can be found in [19].

Lemma 1 For the uniform mobility model, with probability approaching 1 as $n \rightarrow \infty$, the maximum number of nodes in any cell is given by

$$
Z_{\text {max }}=\left\lceil\frac{3 \log (n)}{\log \left(\log \left(n^{\phi}\right)\right)}\right\rceil .
$$

Although $Z_{\text {max }}$ is the maximum number of nodes in any cell, in practice, the number of codes to be used is limited. Thus, at most $\mathcal{A}$ nodes in any cell are allowed to get a code and communicate during $t_{d a t a}$. However, $Z_{\max }$ grows very slowly with $n$. Thus, by choosing, for example, $\mathcal{A} \geq 10$, for practical values of $\phi$, the fraction of cells having more than $\mathcal{A}$ nodes can be bounded by a small constant, for $n$ large. Accordingly, the total number of cells in the network is (\# of cells) $=1 / a(n)=\phi n$. By considering the uniform mobility model, the fraction of cells containing $Z=j$ nodes is obtained by

$\mathbb{P}\{Z=j\}=\left(\begin{array}{c}n \\ j\end{array}\right)\left(\frac{1}{\phi n}\right)^{j}\left(1-\frac{1}{\phi n}\right)^{n-j} \stackrel{n \rightarrow \infty}{\longrightarrow} \frac{1}{j !}\left(\frac{1}{\phi}\right)^{j} e^{-1 / \phi}$.

The fraction of cells having more than $\mathcal{A}$ nodes as $n \rightarrow \infty$, for given $\phi$, can be upper-bounded by

$\mathbb{P}\{Z>\mathcal{A}\}=\sum_{j=\mathcal{A}+1}^{\infty} \frac{1}{j !}\left(\frac{1}{\phi}\right)^{j} e^{-1 / \phi} \leq \frac{1}{(\mathcal{A}+1) !}\left(\frac{1}{\phi}\right)^{\mathcal{A}+1}$.

For example, for $\phi=\frac{1}{3}$ and $\mathcal{A}=10, \mathbb{P}\{Z>\mathcal{A}\} \leq 0.0044$ as $n \rightarrow \infty$.

\section{Interference in a Data Channel}

The interference in the data channel at a node $j$, regarding node $i$ transmitting to node $j$ through $W_{j}$, is defined as the signals coming from all transmitting nodes in the network, via $W_{j}$, except node $i$. It can be decomposed in the following two types.

Destructive Interference (DEI) for the node $j$ comes from nodes, transmitting in $W_{j}$, outside the receiver range of $j . D E I$ constitutes the part of the interference that will not be decoded.

Constructive Interference (COI) comes from nodes, transmitting in $W_{j}$, within the receiver range of $j$. By construction (see Section III-A), the nodes within the receiver range of $j$, transmitting in $W_{j}$, use different codes. $C O I$ constitutes the decodable part of the interference.

If node $i$ transmits data to $j$ at time $t$, via $W_{j}$, the SNIR at the receiver $j$, without SIC, is given by (7) [2], where range is the set of nodes transmitting in $W_{j}$ and reached by the receiver range of node $j, C_{i}$ is the PN sequence used by sender node $i, P_{i j}(t)=P \forall(i, j)$ is the transmit power chosen by

${ }^{8} k \notin$ range means the nodes outside the receiver range of node $j$ transmitting in $W_{j}$. 


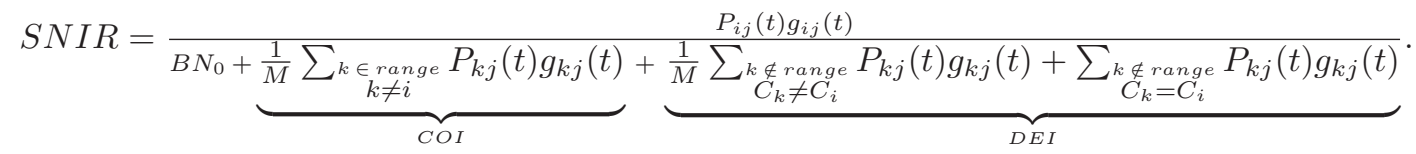

node $i$ to transmit to node $j$ (i.e., $P_{i j}(t)$ is constant for all pair $(i, j)), g_{i j}(t)$ is the channel path gain from node $i$ to $j, B$ is the original bandwidth of the data signal (before spreading), $B N_{0}$ is the noise power (where $N_{0}$ is the noise power spectral density), $M$ is the spreading factor, $C O I$ and $D E I$ are the total interference in $W_{j}$ at node $j$. The summation terms in the denominator of (7) containing the factor $1 / M$ constitute the multiple access interference $(M A I) . M A I$ is caused by partial cross-correlation among the distinct codes due to the asynchronous nature of the uplink channel [7]. The last summation term (without the factor $1 / M$ ) is consequence of code reuse in the network and we call it same code interference $(S C I)$. Thus, $S C I=\sum_{\substack{k \notin \text { range } \\ C_{k}=C_{i}}} P_{k j}(t) g_{k j}(t)$, such that, $M A I+S C I=C O I+D E I . M A I$ and $S C I$ presentations are easier for calculating SNIR as explained later.

The channel path gain $g_{i j}$ is assumed to be a function of the distance only (i.e., the simple path propagation model) [1], [2], therefore, $g_{i j}(t)=\frac{1}{\left|X_{i}(t)-X_{j}(t)\right|^{\alpha}}=\frac{1}{r_{i j}^{\alpha}(t)}$, in which $\alpha$ is the path loss parameter, and $r_{i j}(t)$ is the distance between $i$ and $j$.

\section{Hybrid FDMA/CDMA Data Transceiver}

From Fig. 3(downlink), the FDMA/CDMA data transmitter in node $j$ selects packets previously relayed to node $j$ which have their destination nodes present in the same cell, spread the data using the code $C_{j}$ assigned to node $j$, and transmits each one of them through each different frequencies associated to each distinct destination node. If the node assigned to a data channel is not a destination for a relayed packet, then the transmitter selects a new packet generated locally by node $j$.

The basic decoding scheme of the CDMA-SIC data receiver scheme is given in [6] (see also Fig. 3(uplink)), in which the decoding is performed successively from the strongest signal to the weakest. The use of training sequences obtained through the control channels allow to obtain a local estimation of the wireless channel. Thus, with the simple path propagation model assumed, the strongest signal decoded first comes from the closest neighbor to node $j$ (not necessarily in the same cell of $j$ but in the cluster it perceives), while the weakest (decoded last) is the farthest node to node $j$ in the cell node $j$ is located. Let $M A I^{\prime}$ be the remaining multiple access interference at node $j$ after applying SIC up to node $i$, i.e.,

$$
M A I^{\prime}=\frac{1}{M} \sum_{\substack{\forall k: g_{k j}<g_{i j} \\ C_{k} \neq C_{i}}} P_{k j}(t) g_{k j}(t) .
$$

Therefore, the resulting SNIR (called $S N I R^{\prime}$ ) from node $i$ to node $j$ after applying SIC is given by

$$
S N I R^{\prime}=\frac{P_{i j}(t) g_{i j}(t)}{B N_{0}+M A I^{\prime}+S C I} .
$$

Note that, depending on the position of the node $j$, it may have nodes transmitting from adjacent cells closer than a far node in the same cell. Therefore, $j$ has to be able to decode the data signals from these adjacent cell nodes before decoding the signal from the far node of the same cell. This explains why each node also needs to obtain the training sequences from the other nodes located outside its cell but still within its receiver range. The receiver uses the information obtained during the neighbor discovery phase to retain the data packets from nodes in the same cell as $j$, dropping the outside cell packets since node $j$ cannot keep track of all nodes in adjacent cells to see if this packet is for relaying or destination. Besides, from (7), SIC is fundamental to derive (9) and a node have all packets from the same cell successfully decoded.

\section{CAPACITY AND BANDWidTh ANALYSis}

\section{A. Link's Shannon Capacity}

The link's Shannon capacity $R_{i j}$ in the data channel $W_{j}$, in which node $j$ receives from node $i$, after $j$ applying SIC up to node $i$, from (9), is given (in units of nats) by [20]

$$
R_{i j}=B \log \left(1+\frac{P_{i j}(t) g_{i j}(t)}{B N_{o}+M A I^{\prime}+S C I}\right) .
$$

$M A I^{\prime}$ can be computed by using Fig. 5. Assume that the center of the unit square area is the origin $O$ of the $(x, y)$ coordinates, and that, at time $t$, the receiver node $j$ is located at the point $Q$ with coordinates $\left(x_{Q}, y_{Q}\right) \in\left(-\frac{1}{2}, \frac{1}{2}\right)$. The calculation considers the transmitting node $i$ located at a distance $c_{1} \sqrt{a(n)}$ from $j$, while due to SIC, all the remaining interfering nodes are at a distance greater than $c_{1} \sqrt{a(n)}$ from $j$, where $c_{1} \in(0, \sqrt{2})$ depends on the distance between node $j$ and all other nodes in the cell. We divide the square unit area network in four triangles and compute the interference generated from each of these regions, such that $M A I^{\prime}=\sum_{l=1}^{4} M A I_{l}^{\prime}$. Similar to [4], for a uniform distribution of the nodes, we consider a differential element area $r d r d \gamma$ that is distant $r$ units from node $j$. Since the nodes are uniformly distributed and $n$ grows to infinity, the node density in the network is $\frac{n}{1}$, and the summation in (8) can be bounded by an integral. Thus, $M A I_{l}^{\prime}$ at node $j$ is upper-bounded by

$$
M A I_{l}^{\prime}(n) \leq \int_{r e g i o n} \int_{M A I_{l}^{\prime}} \frac{P}{M r^{\alpha}} \phi \epsilon_{j} \frac{n}{1} r d r d \gamma,
$$

in which $\epsilon_{j}$ is the fraction of cells using the bandwidth $W_{j}$. Accordingly, $\epsilon_{j}$ equals the fraction of cells containing at least $j$ nodes, in which $j \in[2, \mathcal{A}]$. From (5), we have as $n \rightarrow \infty$

$$
\epsilon_{j}=\mathbb{P}\{Z \geq j\}=1-\sum_{k=0}^{j-1} \frac{1}{k !}\left(\frac{1}{\phi}\right)^{k} e^{-1 / \phi} .
$$

Thus, for $\alpha>2$, and using that $a(n)=\frac{1}{\phi n}$, from (11) we obtain with some manipulations

$$
\begin{aligned}
M A I_{l}^{\prime}(n) & \leq \int_{\gamma_{\min _{l}}}^{\gamma_{\max _{l}}} \int_{c_{1} \sqrt{a(n)}}^{r_{\max l}(\gamma)} \frac{P \phi \epsilon_{j} n}{M r^{\alpha-1}} d r d \gamma \\
& \leq c_{2} \epsilon_{j} n^{\frac{\alpha}{2}}\left(1-\frac{c_{3}}{n^{\frac{\alpha}{2}-1}}\right),
\end{aligned}
$$

in which $c_{2}$ and $c_{3}$ are positive constants for given $l,\left(x_{Q}, y_{Q}\right)$, $c_{1}, \phi, M, P$, and $\alpha$. Therefore,

$$
M A I^{\prime}=\sum_{l=1}^{4} M A I_{l}^{\prime} \leq c_{4} \epsilon_{j} n^{\frac{\alpha}{2}} \leq c_{4} n^{\frac{\alpha}{2}},
$$

since $\epsilon_{j} \in[0,1]$, and $\left(1-\frac{c_{3}}{n^{\frac{\alpha}{2}-1}}\right) \leq 1$ for $n$ large. $c_{4}$ is a positive constant function of the location $\left(x_{Q}, y_{Q}\right)$ of node $j$.

On the other hand, the same code interference $(S C I)$ can be upper-bounded by using the same procedure as done before for $M A I^{\prime}$. Consequently, it can be shown that 


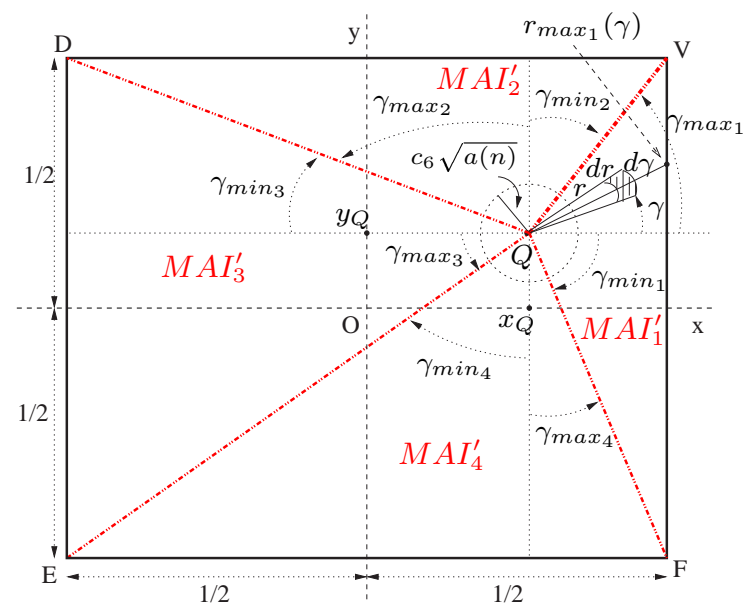

Fig. 5. Interference regions for node $i$ communicating with node $j$. The angle $\gamma$ increases in the counterclockwise direction.

$$
S C I=\sum_{\substack{k \notin \text { range } \\ C_{k}=C_{i}}} P_{k j}(t) g_{k j}(t) \leq c_{5} \epsilon_{j} n^{\frac{\alpha}{2}} \leq c_{5} n^{\frac{\alpha}{2}}
$$

Hence, from (14) and (15), it results that the total remaining interference after SIC at node $j$ is upper-bounded by

$$
M A I^{\prime}+S C I \leq\left(c_{4}+c_{5}\right) n^{\frac{\alpha}{2}} .
$$

If we consider the expansion $B=f(n)$ of the original data bandwidth, ${ }^{9}$ such that $1 \leq f(n)<n^{\frac{\alpha}{2}}$, then, a lower-bound for $R_{i j}$ can be obtained by using the maximum interference. Thus, from (10) and (16), the corresponding link's Shannon capacity lower-bound as $n \rightarrow \infty$, for node $j$ receiving from node $i$, is obtained by

$$
R_{i j} \geq f(n) \underbrace{\log \left(1+\frac{c_{6} n^{\frac{\alpha}{2}}}{f(n) N_{o}+\left(c_{4}+c_{5}\right) n^{\frac{\alpha}{2}}}\right)}_{n \rightarrow \infty}=c_{7} f(n),
$$

in which $c_{6}$ and $c_{7}$ are positive constants for given $\alpha, \phi, P, M$, $N_{o}, c_{1}$, and $\left(x_{Q}, y_{Q}\right)$. In (17), interference dominates noise for the bandwidth expansion $1 \leq B<n^{\frac{\alpha}{2}}$.

On the other hand, if we consider a scenario such that there is no limitation on available bandwidth, then we can obtain an upper-bound for $R_{i j}$. Accordingly, from (10),

$$
R_{i j}=B \log \left(1+\frac{c_{6}}{\frac{B N_{o}}{n^{\frac{\alpha}{2}}}+\frac{1}{n^{\frac{\alpha}{2}}}\left(M A I^{\prime}+S C I\right)}\right) .
$$

Now, from (16) and (18), and by taking $B \geq c_{8} n^{\frac{\alpha}{2}}$, for some positive constant $c_{8}$ and $n$ sufficiently large, it results that

$$
\frac{1}{n^{\frac{\alpha}{2}}}\left(M A I^{\prime}+S C I\right) \leq c_{4}+c_{5} \leq \frac{B N_{o}}{n^{\frac{\alpha}{2}}} .
$$

Thus, the term $\frac{B N_{o}}{n^{\frac{\alpha}{2}}}$ becomes dominant in the denominator of (18) when $B \geq c_{8} n^{\frac{\alpha}{2}}$ and $n \rightarrow \infty$. From (18) and (19), for $B \geq c_{8} n^{\frac{\alpha}{2}}$, we have the following upper-bound for the link's Shannon capacity as $n \rightarrow \infty$

$$
R_{i j}=n^{\frac{\alpha}{2}} \underbrace{\frac{B}{n_{9}} \log \left(1+\frac{c_{6}}{\frac{B N_{o}}{n^{\frac{\alpha}{2}}}+\frac{1}{n^{\frac{\alpha}{2}}}\left(M A I^{\prime}+S C I\right)}\right)}_{\substack{n \rightarrow \infty \\ n^{\frac{\alpha}{2}}}}=c_{9} n^{\frac{\alpha}{2}},
$$

in which $c_{9}$ is a positive constant. Here, noise dominates interference due to the large bandwidth expansion.

\footnotetext{
${ }^{9}$ In our analysis, the bandwidth expansion is used to obtain the asymptotic behavior of the capacity.
}

Thus, (17) and (20) describe two limiting cases. The former is the minimum capacity attained if we use the bandwidth expansion $1 \leq B<n^{\frac{\alpha}{2}}$. The latter is the maximum capacity reachable if the available bandwidth is large such that $B \geq c_{8} n^{\frac{\alpha}{2}}$. Note that any increase in $B$ beyond $c_{8} n^{\frac{\alpha}{2}}$ will not change the order of the upper-bound of the capacity.

\section{B. Per Source-Destination Throughput}

From Section III-B, each node accesses the data channel at a constant rate $\delta=\frac{t_{\text {data }}}{t_{d i s c}+t_{\text {data }}}$ with probability approaching 1 as $n \rightarrow \infty$, such that each source sends one packet per session to its destination. Each node is guaranteed, in each data channel, a communication rate of $R_{i j}$ lower- and upper-bounded by (17) and (20), respectively. Also, this available communication rate has to be divided among all routes the node must serve per session per channel. However, due to the mobility and the routing scheme, each node serves only one route per session per data channel, i.e., the node either relays a new packet or it delivers a packet to a destination. Thus, the number of routes every node has to service per session per data channel is (\# of served routes) $=1$. Moreover, all cells containing at least two nodes are able to execute FDMA/CDMA and SIC successfully. From (5), $\mathbb{P}\{Z \geq 2\}=\left(1-e^{-1 / \phi}-\frac{1}{\phi} e^{-1 / \phi}\right)$, as $n \rightarrow \infty$. Hence, with probability approaching 1 as $n \rightarrow \infty$, the per source-destination throughput $\lambda(n)$ is obtained by [14], [4]

$$
\lambda(n)=\frac{R_{i j} \delta \mathbb{P}\{Z \geq 2\}}{\# \text { of served routes }}=c_{10} R_{i j},
$$

where $c_{10}$ is a positive constant for given $t_{d i s c}, t_{d a t a}$, and $\phi$. From (17), (20), and (21), we proved the following theorem.

Theorem 1 By employing mobility, CDMA, SIC, one-time relaying of packets, and bandwidth expansion using the manyto-many cooperation strategy, the ad hoc network attains, with probability approaching 1 as $n \rightarrow \infty$, the upper-and lowerbound per source-destination throughput given respectively by

$$
\lambda(n)=O\left(n^{\frac{\alpha}{2}}\right) \text { and } \lambda(n)=\Omega[f(n)],
$$

where $1 \leq f(n)<n^{\frac{\alpha}{2}}$.

\section{Bandwidth Scalability}

The total bandwidth requirement $\left(\Delta W_{\text {total }}\right)$ for the entire network has two components. One from the control channels $\left(\Delta \omega_{C}\right)$, and the other from the data channels $\left(\Delta W_{D}\right)$.

From (2) and (3), Lemma 1 , and noting that $\Delta \omega$ in each control channel equals $2 / T$, due to the Nyquist rate, it results that

$$
\Delta \omega_{C}=\frac{24 N_{\min }}{t_{d i s c}}=\Theta\left[1-\left(1-\frac{\log (\log (n))}{\log (n)}\right)\left[\frac{1}{\left[\frac{3 \log (n)}{\log \left(\log \left(n^{\phi}\right)\right)}\right]}\right]^{-1} .\right.
$$

From Section III-A, $\Delta W=B M=12 \mathcal{A} B$ [7]. Thus, the bandwidth scalability in each data channel associated to the upper- and lower-bound capacity is given respectively by

$$
\Delta W=\Omega\left(n^{\frac{\alpha}{2}}\right) \text { and } \Delta W=\Theta[f(n)],
$$

where $1 \leq f(n)<n^{\frac{\alpha}{2}}$.

The total bandwidth for the entire network is obtained by

$$
\Delta W_{\text {total }}=\Delta W_{D}+\Delta \omega_{C}=\mathcal{A} \Delta W+\Delta \omega_{C},
$$

where $\Delta W$ and $\Delta \omega_{C}$ are given above. 


\section{Comparison With Previous Schemes}

\section{A. The Static Network Case}

Here, we consider the capacity and bandwidth expansion performance of the CDMA-SIC MAC layer scheme assuming a static network, and compare it with the results by Negi and Rajeswaran [11], who employed bandwidth expansion in the model presented by Gupta and Kumar [1].

The model we consider is that of a network formed by $n$ fixed nodes, independently and uniformly distributed on a unit sphere surface. This model is also known as random network [1]. The set of assumptions assumed here are basically the same as those adopted in the previous sections, except that the $n$ nodes are considered to be static on a unit sphere surface, and that the routing of packets is done through multiple hops along cells following the minimum distance path from source to destination. These assumptions are also consistent with the works by Negi and Rajeswaran [11] and Gupta and Kumar [1]. Because the communication framework is one-to-one, we use only one data channel $\Delta W$ for the entire network. Therefore, no simultaneous data channels are needed given that only one copy of each packet is relayed along the route to its destination, and so we can apply the CDMA-SIC without FDMA.

In this model, the surface of the sphere is divided into cells. The number of nodes in any cell is a random variable $Z$. A node is randomly chosen to relay all traffic in each cell, and is called the head node of the cell. Thus, to guarantee relaying of traffic between cells, it is required that every cell has at least one node whp [1], i.e., $\mathbb{P}\{Z \geq 1\} \stackrel{n \rightarrow \infty}{\longrightarrow} 1$. Within a cell, all sources send traffic to the head node, and destinations receive traffic from the head node.

Gupta and Kumar [1] showed that there exists a Voronoi tessellation $\mathcal{V}_{n}$ on the unit sphere surface satisfying the following properties:

- Every Voronoi cell $V$ contains a disk of area $100 \log (n) / n$ and corresponding radius $\rho(n)=$ $c_{11} \sqrt{\log (n) / n}$.

- Every Voronoi cell is contained within a circle of radius $2 \rho(n)$.

Each Voronoi cell $V \in \mathcal{V}_{n}$ is simply a cell of the network, and the cells do not have a regular shape because the network is random. With this tessellation, each cell contains at least one node $w h p$ which meets the connectivity requirement [1]. Furthermore, by choosing the transmission range equal to $8 \rho(n)$ for each node, it allows direct communication within a cell and between adjacent cells. Accordingly, two cells are interfering neighbors if there is a point in one cell that is within a distance $(2+\Delta) 8 \rho(n)$ of some point in the other cell, in which $\Delta>0$ is a given constant modeling situations where a guard zone is required to prevent a neighboring node from transmitting on the same channel at the same time [1].

Another useful property of this Voronoi tessellation is that every cell $V \in \mathcal{V}_{n}$ has no more than $c_{12}$ interfering neighbors, and hence the maximum number of interfering nodes is bounded by some positive constant [1]. Consequently, similarly to what we did in the mobile case, we can assign distinct PN sequences to each node, such that every cell in $\mathcal{V}_{n}$ has interfering neighbors using different codes. Therefore, we need $M \geq c_{12}$ distinct PN sequences and reuse the codes in order to save bandwidth. Note that GPS (or some other technique) is no longer required since nodes are static. However, as explained before, MAI has to be considered even when transmission synchronization among nodes is employed [7]. Because nodes are static, we only need to assign the different codes during the initialization of the network.

We compute the link's Shannon capacity for an arbitrary pair of nodes from adjacent cells, noting that the analysis applied for the mobile network can be used for the static network as well. Thus, similarly to the description in Section III-C, each node communicating with another node applies SIC to eliminate MAI from close neighbors and the $S N I R^{\prime}$ computation follows (9). The MAI calculation is done following an approach similar to that of (13), but considering the unit sphere surface. Furthermore, because the communication is either between two nodes on the same cell or between two head nodes from adjacent cells, any two communicating nodes are located at distance $c_{13} \rho(n)$ apart from each other. Therefore, if node $j$ is receiving data from node $i$, after node $j$ applies SIC up to node $i$, all the remaining interfering nodes are placed at distance greater than $c_{13} \rho(n)$. Hence, for $\alpha>2$ and $n$ sufficiently large, we have the following bound for the remaining multiple access interference at a node $j$ receiving data from node $i$, after SIC,

$$
\begin{aligned}
M A I^{\prime}(n) & \leq \int_{0}^{2 \pi} \int_{c_{13} \rho(n)}^{\frac{\sqrt{\pi}}{2}} \frac{P \phi n}{M r^{\alpha-1}} d r d \gamma \\
& =\frac{2 \pi P \phi n}{M(\alpha-2)}\left[\left(\frac{n}{c_{14} \log (n)}\right)^{\frac{\alpha}{2}-1}-\left(\frac{2}{\sqrt{\pi}}\right)^{\alpha-2}\right] \\
& \leq \frac{c_{15} n^{\frac{\alpha}{2}}}{(\log (n))^{\frac{\alpha}{2}-1}}
\end{aligned}
$$

where $c_{15}$ is a positive constant given that $c_{11}, c_{13}, \phi, M, P$, and $\alpha$ are specified.

Analogously, the same code interference $(S C I)$ can be upper-bounded by

$$
S C I=\sum_{\substack{k \notin \text { range } \\ C_{k}=C_{i}}} P_{k j}(t) g_{k j}(t) \leq \frac{c_{16} n^{\frac{\alpha}{2}}}{(\log (n))^{\frac{\alpha}{2}-1}} .
$$

Hence, from (26) and (27), the total remaining interference after SIC at node $j$ is upper-bounded by

$$
M A I^{\prime}+S C I \leq\left(c_{15}+c_{16}\right) \frac{n^{\frac{\alpha}{2}}}{(\log (n))^{\frac{\alpha}{2}-1}} .
$$

From (10), assuming that node $i$ transmits to $j$, in which the original data bandwidth is $B$ (before spreading), we obtain the following link's Shannon capacity

$$
\begin{aligned}
R_{i j} & =B \log \left(1+\frac{\frac{P}{\left(c_{13} \rho(n)\right)^{\alpha}}}{B N_{o}+M A I^{\prime}+S C I}\right) \\
& =B \log \left(1+\frac{c_{17}}{\frac{B N_{o}(\log (n))^{\frac{\alpha}{2}}}{n^{\frac{\alpha}{2}}}+\frac{(\log (n))^{\frac{\alpha}{2}}}{n^{\frac{\alpha}{2}}}\left(M A I^{\prime}+S C I\right)}\right) .
\end{aligned}
$$

For the term associated with the maximum interference over the unit sphere surface, we have from (28) that

$$
\frac{(\log (n))^{\frac{\alpha}{2}}}{n^{\frac{\alpha}{2}}}\left(M A I^{\prime}+S C I\right) \leq \log (n)\left(c_{15}+c_{16}\right) .
$$

Thus, from (29) and (30), and by taking $B \geq \frac{c_{18} n^{\frac{\alpha}{2}}}{(\log (n))^{\frac{\alpha}{2}-1}}$, for some positive constant $c_{18}$ and $n$ sufficiently large, we obtain

$$
\begin{aligned}
\frac{(\log (n))^{\frac{\alpha}{2}}}{n^{\frac{\alpha}{2}}}\left(M A I^{\prime}+S C I\right) & \leq \log (n)\left(c_{15}+c_{16}\right) \\
& \leq \frac{B N_{o}(\log (n))^{\frac{\alpha}{2}}}{n^{\frac{\alpha}{2}}} .
\end{aligned}
$$


The term $\frac{B N_{o}(\log (n))^{\frac{\alpha}{2}}}{n^{\frac{\alpha}{2}}}$ becomes dominant in the denominator of (29) when $B \geq \frac{c_{18} n^{\frac{\alpha}{2}}}{(\log (n))^{\frac{\alpha}{2}-1}}$ and $n \rightarrow \infty$. Consequently, for $B \geq \frac{c_{18} n^{\frac{\alpha}{2}}}{(\log (n))^{\frac{\alpha}{2}-1}}$, the link's Shannon capacity as $n \rightarrow \infty$ is given by (32) (see top of next page) in which $c_{19}$ is a positive constant.

Eq. (32) is the link's Shannon capacity obtained from the noise dominance over interference due to large bandwidth expansion. Note that any increase in $B$ beyond $\frac{c_{18} n^{\frac{\alpha}{2}}}{(\log (n))^{\frac{\alpha}{2}-1}}$ does not change the value of this capacity.

The bandwidth expansion associated to this capacity, in which $B \geq \frac{c_{18} n^{\frac{\alpha}{2}}}{(\log (n))^{\frac{\alpha}{2}-1}}$, is given by

$$
\Delta W=B M=\Omega\left[\frac{n^{\frac{\alpha}{2}}}{(\log (n))^{\frac{\alpha}{2}-1}}\right] .
$$

To obtain the throughput behavior, note that each cell has one node $w h p$, and any node in this cell can be the head node to relay all the traffic the cell must handle, while the other nodes can simply serve as sources or destinations. Accordingly, analogous to (21), the per source-destination throughput is given $w h p$ by

$$
\lambda(n)=\frac{R_{i j} \delta \mathbb{P}\{Z \geq 1\}}{\# \text { of served routes }},
$$

where $\mathbb{P}\{Z \geq 1\} \stackrel{n \rightarrow \infty}{\longrightarrow} 1$, and $\delta$ is a constant that depends on $c_{12}$ and can be computed based on the channel access scheme employed [1].

The number of routes served by any cell is a consequence of the routing strategy. As mentioned before, the routing of packets is done through multiple hops along cells following the minimum distance path from source to destination, i.e., every packet follows the straight line segment connecting the source to its destination. Therefore, the traffic to be carried by any cell is proportional to the number of straight line segments passing through the cell. Accordingly, the number of routes intersecting any cell is bounded by the following lemma, which was proved by Gupta and Kumar [1].

Lemma 2 The total number of source-destination lines (i.e., routes) intersecting every cell in the random network can be bounded whp by

$\sup _{V \in \mathcal{V}}($ Number of routes intersecting $V) \leq c_{20} \sqrt{n \log (n)}$

Therefore, using the network model assumptions provided in this Section, from (32) and (34), and from Lemma 2, we proved the following Theorem.

Theorem 2 The static random wireless ad hoc network using CDMA and SIC attains whp the following per sourcedestination throughput lower-bound

$$
\lambda(n)=\Omega\left[\frac{n^{\frac{\alpha-1}{2}}}{(\log (n))^{\frac{\alpha+1}{2}}}\right] .
$$

Theorem 2 provides the same throughput lower-bound order as that obtained by Negi and Rajeswaran [11], which corroborates the capacity analysis technique employed throughout this paper. However, our bandwidth expansion associated to this lower-bound, given by (33), is much smaller than the $\Theta\left(n\left(n^{2} \log (n)\right)^{\frac{\alpha}{2}}\right)$ required by Negi and Rajeswaran [11] because we take advantage of SIC. SIC allows every node in the network to successfully receive the packets from its close neighbors increasing the minimum distance of the destructive interferers. In our case, the closest destructive interferer is located whp at distance $\Omega(\rho(n))=\Omega(\sqrt{\log (n) / n})$ due to SIC, while in [11] this distance is $\Omega(1 / n \sqrt{\log (n)})$ whp.

The strategies used to obtain the throughput lower-bound here and in [11] assume that each node transmits at constant power and its packets follow the minimum distance path to the destination. However, it was also shown by Negi and Rajeswaran [11] that, if transmission power control is allowed, then a minimum power route to destination can be obtained (not necessarily equal to the minimum distance path) which provides an upper-bound for the throughput. It is the objective of our future research to allow power control in our analysis and investigate the associated behavior of the throughput and bandwidth expansion considering CDMA and SIC.

\section{B. The Mobile Network Case}

A direct comparison between many-to-many cooperation and the strategy proposed by Grossglauser and Tse [2] is not appropriate even after applying CDMA and bandwidth expansion, because their model does not require the use of cell we assume to enable frequency reuse. Accordingly, we extend Grossglauser and Tse's network model by introducing cells in which nodes are endowed with FDMA/CDMA-SIC and GPS capabilities, such that every node behaves simultaneously like sender and receiver of data packets for each communication session. Therefore, another comparison, not necessarily based on the physical layer properties (like link's Shannon capacity or bandwidth expansion), is more suitable.

We have recently presented a cell description [4] for Grossglauser and Tse's scheme [2] using assumptions that are similar to those used by El Gamal et al. [14]. Because only one halfduplex data channel is used for the entire network in Grossglauser and Tse's model [2], a node cannot be sender and receiver simultaneously, but rather every node behaves like either a sender or a receiver for each communication session. Accordingly, $r_{o}=1 / \sqrt{\pi \theta n}$ determines a cell in such a model [2], [4] for a uniform distribution of the nodes, where the parameter $\theta \in(0,1)$ is defined as the fraction of sender nodes $n_{S}$ in the network. Therefore, $n_{S}=\theta n$, and $n_{R}=(1-\theta) n$ is the fraction of receiver nodes. It has been shown [14], [4] that the per source-destination throughput is proportional to the fraction of cells in the network that can successfully forward packets. In the work by Grossglauser and Tse [2] and in our previous work [4], only the cells containing exactly one sender (i.e., $L=1$ ) and at least one receiver (i.e., $K \geq 1$ ) are able to forward packets, because no SIC capability is assumed, and therefore, the cells containing more than one sender present transmission collisions, preventing successful relaying of packets. Similarly to what was done to obtain (5), it can be shown that, for Grossglauser and Tse's scheme [2], we have that as $n \rightarrow \infty$ [4]

$$
\mathbb{P}\{L=1, K \geq 1\}=\frac{1}{\theta} e^{-1 / \theta}\left(1-e^{-1 / \theta}\right) .
$$

With many-to-many cooperation, in order to obtain the same cell size as in [2] and [4], i.e, $a(n)=\pi r_{o}^{2}=\frac{1}{\theta n}=\frac{1}{\phi n}$, we must set $\theta=\phi$, and use a finite bandwidth expansion. In addition, all cells containing at least two nodes are able to successfully forward packets in many-to-many cooperation. Thus, from (5), $\mathbb{P}\{Z \geq 2\}=\left(1-e^{-1 / \phi}-\frac{1}{\phi} e^{-1 / \phi}\right)$ as $n \rightarrow \infty$. Hence, 


$$
\begin{aligned}
R_{i j} & =B \log \left(1+\frac{c_{17}}{\frac{B N_{o}(\log (n))^{\frac{\alpha}{2}}}{n^{\frac{\alpha}{2}}}+\frac{(\log (n))^{\frac{\alpha}{2}}}{n^{\frac{\alpha}{2}}}\left(M A I^{\prime}+S C I\right)}\right) \\
& =\frac{n^{\frac{\alpha}{2}}}{(\log (n))^{\frac{\alpha}{2}}} \underbrace{\frac{n^{\frac{\alpha}{2}}}{(\log (n))^{\frac{\alpha}{2}}}}_{\stackrel{B}{\frac{n^{\frac{\alpha}{2}}}{(\log (n))^{\frac{\alpha}{2}}}} \log \left(1+\frac{c_{17}}{\frac{B N_{o}(\log (n))^{\frac{\alpha}{2}}}{n^{\frac{\alpha}{2}}}+\frac{(\log (n))^{\frac{\alpha}{2}}}{n^{\frac{\alpha}{2}}}\left(M A I^{\prime}+S C I\right)}\right)} .
\end{aligned}
$$

our collaboration-driven strategy provides the following performance gain $G$ over the Grossglauser and Tse's scheme [2] based on a comparison of the fraction of cells that successfully forward packets as $n \rightarrow \infty$,

$$
G=\frac{\mathbb{P}\{Z \geq 2\}}{\mathbb{P}\{L=1, K \geq 1\}}=\frac{1-e^{-1 / \phi}-\frac{1}{\phi} e^{-1 / \phi}}{\frac{1}{\phi} e^{-1 / \phi}\left(1-e^{-1 / \phi}\right)} .
$$

Fig. 6 illustrates the behavior of the gain $G$ given in (38) as a function of $\phi$. Note that $G>1 \forall \phi \in(0,1)$. This gain shows that the throughput is improved by a constant factor compared to the results in [2] and [4] under similar bandwidth expansion. There is additional gain in the link Shannon capacity, as a constant gain factor, due to the use of SIC and the improvement in SNIR. However, an exact computation of this constant factor turns out to be a tedious task.

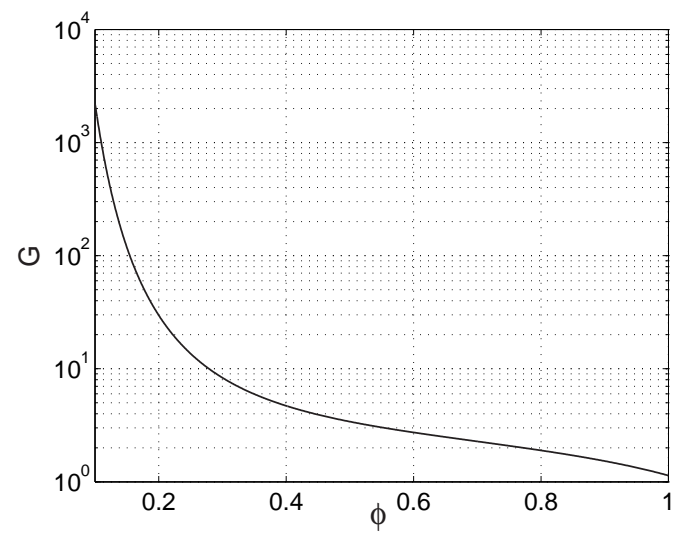

Fig. 6. Performance gain $(G)$ for fraction of cells that successfully forward packets in many-to-many cooperation compared to Grossglauser and Tse's scheme [2].

\section{CONCLUSIONS}

It was shown that the Shannon capacity and per sourcedestination throughput can increase in wireless ad hoc networks by employing mobility, FDMA/CDMA, SIC, and onetime relaying of packets taking advantage of many-to-many cooperation among nodes. Such performance is attained by using successive interference cancellation and distinct codes among close neighbors, which is enabled by running a simple neighbor-discovery protocol. Accordingly, interference from close neighbors is no longer harmful, but rather endowed with valuable data. Also, because multi-copy relaying of packets is employed, the delay performance is improved and follows the description given in [4]. The overall improvement in the network performance is obtained at a cost of increased processing complexity in the nodes. Furthermore, the principles of manyto-many cooperation are applied to static [1], [11] and mobile [2], [4] networks. It is shown that, by using this approach, similar capacity of [11] can be attained with much smaller bandwidth expansion. We have shown that many-to-many cooperation improves the throughput of mobile wireless networks by a constant factor compared to the results in [2] and [4] under similar bandwidth expansion.

\section{ACKNOWLEDGMENT}

This work was supported in part CAPES/Brazil, by the US Army Research Office under grant W911NF-05-1-0246 and by the Basking Chair of Computer Engineering. Opinion, interpretations, conclusions and recommendations are those of the authors and are not necessarily endorsed by the Department of Defense.

\section{REFERENCES}

[1] P. Gupta and P. R. Kumar, "The capacity of wireless networks," IEEE Trans. on Information Theory, vol. 46, no. 2, pp. 388-404, March 2000.

[2] M. Grossglauser and D. Tse, "Mobility increases the capacity of wireless ad-hoc networks," in Proc. of IEEE Infocom, Anchorage, AK, March 2001.

[3] N. Bansal and Z. Liu, "Capacity, delay and mobility in wireless ad-hoc networks," in Proc. of IEEE Infocom, San Francisco, CA, March 2003.

[4] R. M. de Moraes, H. R. Sadjadpour, and J. J. Garcia-Luna-Aceves, "Throughput-delay analysis of mobile ad-hoc networks with a multi-copy relaying strategy," in Proc. of IEEE SECON, Santa Clara, CA, October 2004.

[5] S. Toumpis and A. J. Goldsmith, "Capacity regions for wireless ad hoc networks," IEEE Trans. on Wireless Communications, vol. 2, no. 4, pp. 736-748, July 2003.

[6] P. Patel and J. Holtzman, "Analysis of a simple successive interference cancellation scheme in a DS/CDMA system," IEEE Journal on Selected Areas in Communications, vol. 12, no. 5, pp. 796-807, June 1994.

[7] L. Hanzo, L.-L. Yang, E.-L. Kuan, and K. Yen, Single-and Multi-Carrier DS-CDMA Multi-User Detection, Space-Time Spreading, Synchronisation, Networking and Standards. John Wiley \& Sons, 2003.

[8] B. W. Parkingson and J. J. Spilker, Global Positioning System: Theory and Applications Vol. I. American Institute of Aeronautics and Astronautics, Inc., 1996.

[9] RFR3300 RF-to-IF Receiver, QUALCOMM Inc., August 2004.

[10] V. Rodoplu and T. H. Meng, "Position based CDMA with multiuser detection (P-CDMA/MUD) for wireless ad hoc networks," in Proc. of IEEE Int. Symp. on Spread-Spectrum Tech. \& Appli., NJ, September 2000.

[11] R. Negi and A. Rajeswaran, "Capacity of power constrained ad-hoc networks," in Proc. of IEEE Infocom, Hong Kong, March 2004.

[12] A. Muqattash and M. Krunz, "CDMA-based MAC protocol for wireless ad hoc networks," in Proc. of ACM MobiHoc, Annapolis, MD, June 2003.

[13] A. Hasan, K. Yang, and J. G. Andrews, "High-capacity CDMA ad hoc networks without closed-loop power control," in Proc. of IEEE MILCOM, Boston, MA, October 2003.

[14] A. E. Gamal, J. Mammen, B. Prabhakar, and D. Shah, "Throughput-delay trade-off in wireless networks," in Proc. of IEEE Infocom, Hong Kong, March 2004.

[15] S. Ghez, S. Verdu, and S. Schwartz, "Optimal decentralized control in the random access multipacket channel," IEEE Trans. on Automatic Control, vol. 34, no. 11, pp. 1153-1163, November 1989.

[16] L. Tong, Q. Zhao, and G. Mergen, "Multipacket reception in random access wireless networks: From signal processing to optimal medium access control," IEEE Comm. Magazine, pp. 108-112, November 2001.

[17] R. Knopp and P. A. Humblet, "Information capacity and power control in single-cell multiuser communications," in Proc. of IEEE ICC, Seattle, WA, June 1995.

[18] P. D. L. Beasley, A. G. Stove, B. J. Reits, and B.-O. As, "Solving the problems of a single antenna frequency modulated cw radar," Record of the IEEE 1990 International Radar Conference, pp. 391-395, May 1990.

[19] R. Motwani and P. Raghavan, Randomized Algorithms. Cambridge Univ. Press, 1995.

[20] T. M. Cover and J. A. Thomas, Elements of Information Theory. John Wiley \& Sons, 1991. 